\title{
Optimization of tubing method of biopsy samples during preimplantation genetic testing
}

\author{
Shuai Lii ${ }^{A-D, F}$, Zhiheng Chen ${ }^{A, F}$, Yunhao Liang ${ }^{B, F}$, Hui Wang ${ }^{C, F}$, Li Yang ${ }^{C, F}$, Ling Sun ${ }^{A-D, F}$ \\ Center of Reproductive Medicine, Guangzhou Women and Children's Medical Center, Guangzhou Medical University, China \\ A - research concept and design; B - collection and/or assembly of data; C - data analysis and interpretation; \\ $D$ - writing the article; $E$ - critical revision of the article; $F$ - final approval of the article
}

\section{Address for correspondence}

Ling Sun

E-mail: sunling6299@163.com

\section{Funding sources}

This study was supported by the project grant from the Health Science and Technology Project of Guangzhou (grant No. 20191A011027) and Clinical Research Project of Chinese Medical Association (grant No. 18010210750).

\section{Conflict of interest}

None declared

Received on May 29, 2020

Reviewed on August 10, 2020

Accepted on September 20, 2020

\begin{abstract}
Background. Preimplantation genetic testing (PGT) is a powerful tool for patients with a high risk of transmitting a genetic abnormality to their children. Unlike other assisted reproductive technologies (ART), it has technical issues which remain unresolved.
\end{abstract}

Objectives. To develop a modified tubing method for placing biopsied samples into amplification tubes for PGT.

Material and methods. A modified tubing method was developed and applied to PGT, with the micromanipulator aiding in the fine movement of transfer pipettes, and with a microinjector to minimize the amount of medium which is transferred with the biopsy samples into the amplification tube. A total of 826 blastocysts from 222 PGT cycles performed between December 2016 and December 2019 were retrospectively analyzed. As the tubing of the cells could not always be inspected visually and they would only be detected by the presence of DNA after amplification, the main outcome measure was the amplification success rate.

Results. The amplification success rate with the modified tubing method was $99.6 \%$.

Conclusions. The modified tubing method is efficient and simple. It is a promising technique for PGT tubing. To the best of our knowledge, this is the first report on the use of a modified micromanipulator and microinjector for improving the tubing rate in PGT cycles, and the presented method is by far the closest to actual use for PGT tubing.

Key words: trophectoderm biopsy, preimplantation genetic testing, modified tubing method, vision-based tubing system, biopsy tubing

Cite as

Liu S, Chen Z, Liang Y, Wang H, Yang L, Sun L. Optimization of tubing method of biopsy samples during preimplantation genetic testing. Adv Clin Exp Med. 2020;29(11):1355-1361. doi:0.17219/acem/127679

DOI

10.17219/acem/127679

Copyright

Copyright by Author(s)

This is an article distributed under the terms of the

Creative Commons Attribution 3.0 Unported (CC BY 3.0)

(https://creativecommons.org/licenses/by/3.0/) 


\section{Introduction}

Preimplantation genetic testing (PGT) is used in early prenatal genetic diagnosis, in which abnormal embryos are identified, so that only genetically normal embryos can be used for transfer. Its indications have also been significantly extended to include common late-onset disorders and non-genetic conditions, such as testing for human leukocyte antigens (HLA) matching. ${ }^{1,2}$ There is no doubt that PGT is a powerful tool for patients with a high risk of transmitting a genetic abnormality to their children, though the technique has its own set of specific technical issues. ${ }^{3,4}$

Preimplantation genetic testing requires multiple steps and manipulations of the gametes and embryos in order to select unaffected embryos for transfer and subsequent potential pregnancy. For example, the quantitative polymerase chain reaction (qPCR)-based trophectoderm biopsy for both monogenic diseases and chromosomal abnormalities consists of 32 steps in the process. ${ }^{5}$ The main steps involved in PGT are as follows: embryo biopsy, biopsy tubing, embryo cryopreservation, diagnosis, embryo warming, and embryo transfer. ${ }^{6}$ Placing biopsied samples into amplification tubes (tubing) without losing genetic material is a prerequisite for the ultimate success of DNA amplification. ${ }^{7}$ Cases of DNA amplification failure are mainly due to losing the biopsy samples during the tubing process, which requires repeated moving and washing. ${ }^{8}$ Because of these losses, there may be no biopsy samples to diagnose, which is an outcome that is unacceptable for all couples. ${ }^{9}$

Unfortunately, the conventional method relies on the manual control of micropipettes for transferring embryonic cells to PCR tubes with or without microscopic visualization. ${ }^{10}$ Transferring biopsy samples into PCR tubes and precisely controlling the position of the cells within the micropipette is challenging because of the small volume and the strong adhesion force of the cells involved in the process. Due to the inherently limited manipulability offered by manual control, the outcome is heavily dependent on the operator's ability and experience. These limitations cause the need for optimization of the tubing process.

In recent years, we have seen progress in automation and novel techniques in the field of assisted reproductive technology (ART) manipulation, but there is little or no debate about the optimization of tubing techniques for placing biopsy samples into amplification tubes for PGT. ${ }^{11-14}$ The purpose of this study was to describe a new method for biopsy sample tubing, which allows the tubing step to be precisely controlled, and minimizes the amount of medium transferred along with the cell into the PCR tube. To our knowledge, this is the first report on the use of a modified micromanipulator and microinjector for improving the tubing rate in PGT cycles, and the presented method is by far the closest to the actual use for PGT tubing.

\section{Material and methods}

\section{Patients}

This retrospective study was performed at a single in vitro fertilization (IVF) center and analyzed the PGT cycles of patients who had undergone treatment between December 2016 and December 2019. A total of 826 embryos from 139 patients were biopsied at the blastocyst stage. Only vitrified-warmed single-embryo transfers (SETs) were performed in this study. The primary outcome in this study was the amplification success rate, as the tubing of the cells could occasionally not be inspected visually and would only be detected through the presence of DNA after amplification. The study was approved by the Ethics Committee of Guangzhou Women's and Children's Hospital (China) and informed consent was obtained from all participants.

\section{Embryo culture and biopsy}

Only zygotes presenting with 2 pronuclear bodies were cultured in G1-PLUS/G2-PLUS sequential media (Vitrolife, Gothenburg, Sweden) until they reached the blastocyst stage. Blastocyst grading was performed based on the Gardner and Schoolcraft criteria. ${ }^{15}$ Biopsies were performed on day 5 or day 6 according to the blastocyst grade on that day.

\section{Preparation of modified transfer device}

The modified transfer device assembly consists of a modified micromanipulator, a modified microinjector with a drawn Pasteur pipette, and a homemade PCR rack (Fig. 1A). It was used in conjunction with a stereomicroscope (Fig. 1B). The micromanipulator modified for the movement of the transfer micropipette could be made by dismantling part of the injection holder from a commercial micromanipulator, such as RI (Falmouth, UK), Narishige (Tokyo, Japan), or Eppendorf. The modified microinjector (Fig. 2A) was made from a commercial microinjector, where the end cap on the top of the micropipette holder was replaced with a flexible silica gel connector with an inner diameter of 200$300 \mu \mathrm{m}$. The rack for the PCR tube (Fig. 2B) was made from a petri dish with a notch attached to it to give it a suitable tilting angle $\left(30-35^{\circ}\right)$ for the tube. The micropipettes used to transfer the biopsy samples (Fig. 2A), with an internal diameter of 30-40 $\mu \mathrm{m}$, were drawn using Pasteur pipettes. After being drawn, the glass pipettes were sterilized using dry heat at $160^{\circ} \mathrm{C}$ for $2 \mathrm{~h}$.

\section{Biopsy tubing}

At any step from biopsy tubing onwards, the procedure of moving biopsy samples from the biopsy dish to a PCR tube was performed inside an IVF workstation, with the aid of a modified transfer device and 

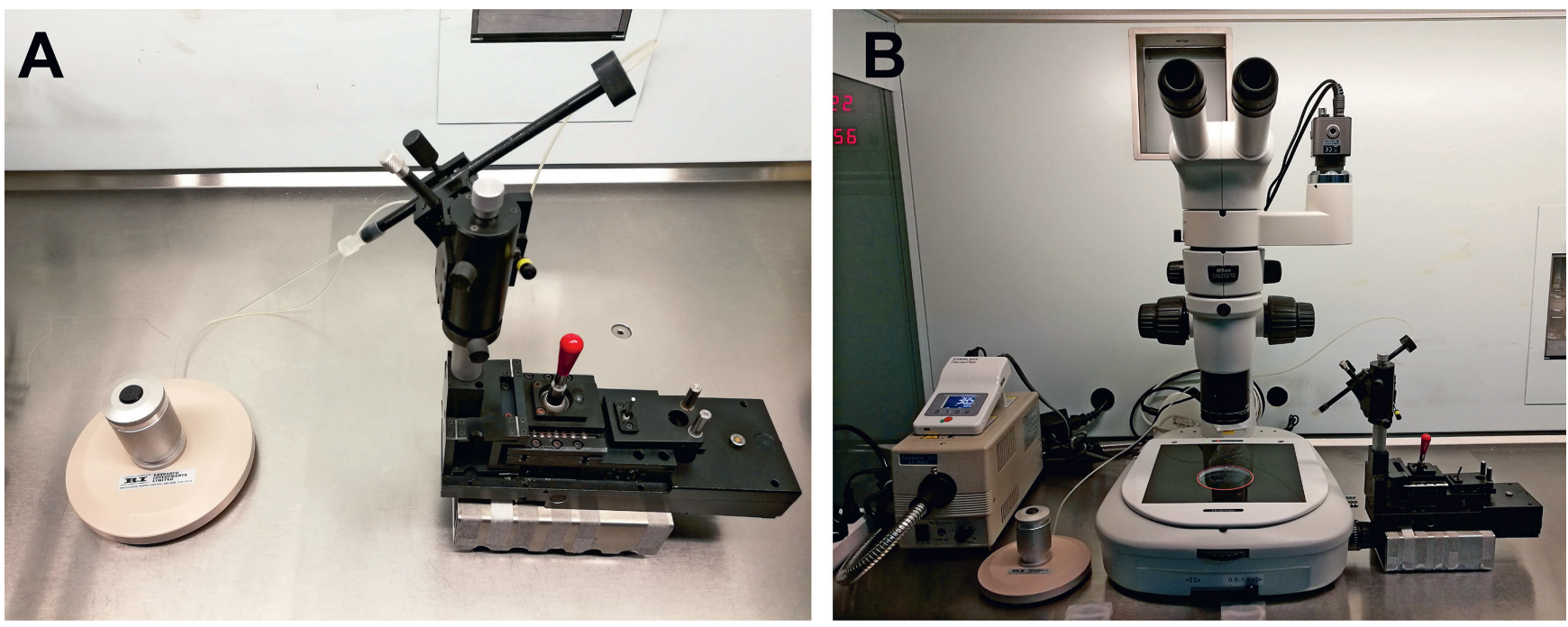

Fig. 1. Representation of the modified transfer device. A. The modified transfer device assembly. B. The modified transfer device in conjunction with stereomicroscope
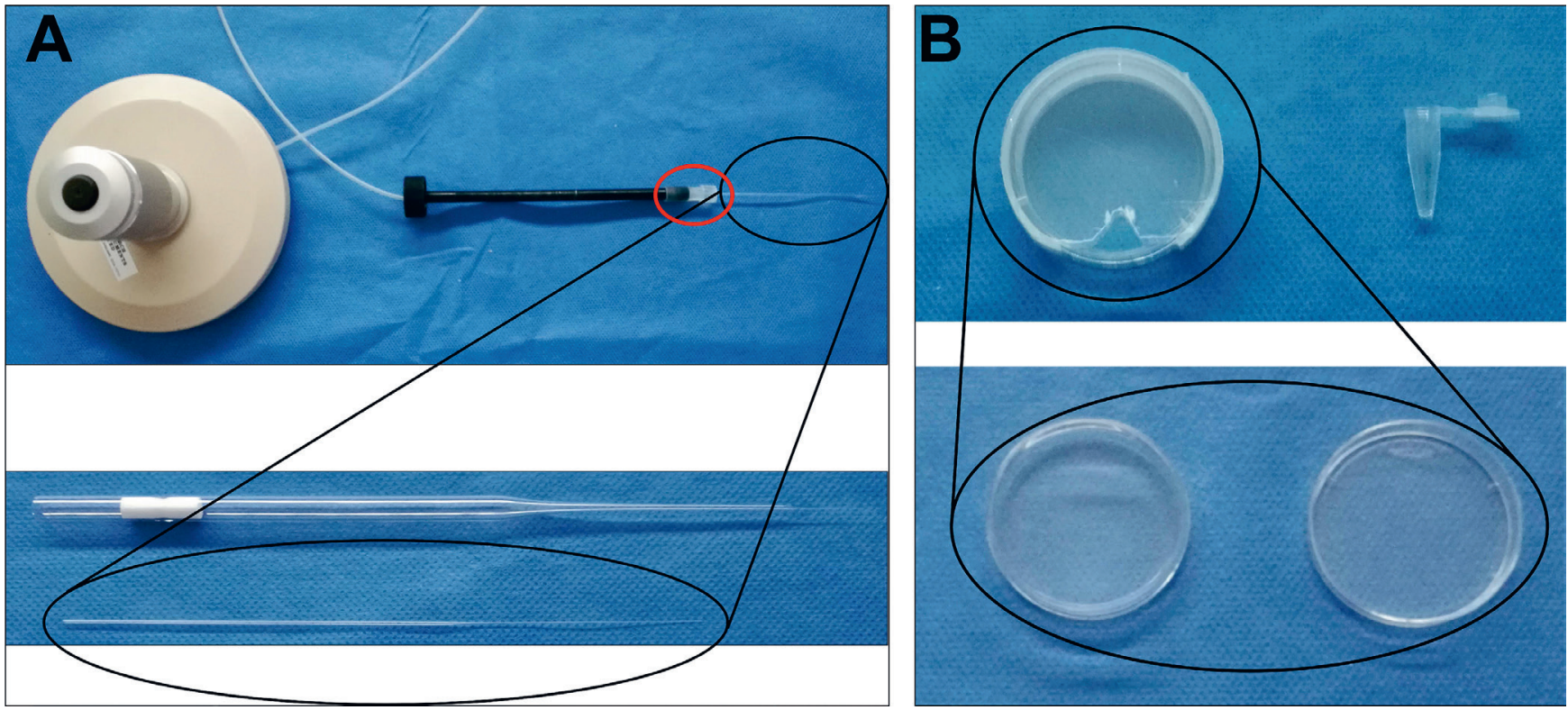

Fig. 2. Material source and construction of modified microinjector, micropipette and PCR tube rack. Inside the red oval is the flexible silica gel connector

a stereomicroscope. In brief, the biopsy dish is moved and placed under the stereomicroscope. The magnification of the stereoscope is adjusted according to the micropipette. With the aid of the modified micromanipulator and microinjector, the transfer micropipette is moved to the biopsy sample, and then the biopsy sample is drawn into the micropipette (Fig. 3A,B). The biopsied specimen is washed with a few drops of clean media to remove paraffin oil, partial sets of chromosomes and other potential contaminants. Following the same steps, the biopsy samples in the micropipette are moved into the corresponding PCR tube (Fig. 3C,D).

To avoid the biopsied cells becoming stuck to the inner wall of the transfer micropipette, the micropipette should be washed in $7 \%$ polyvinylpyrrolidone (PVP) medium before biopsy tubing.

\section{Statistical analysis}

All data analysis was performed using SPSS Statistics v. 25 software (IBM Corp., Armonk, USA). The statistics are expressed as means \pm standard deviation (SD) for continuous variables, while percentages are used for categorical variables.

\section{Results}

\section{Characteristics and embryological data of the study patients}

A total of 826 blastocyst-stage biopsies from 222 PGT cycles at a single center using the modified tubing method 

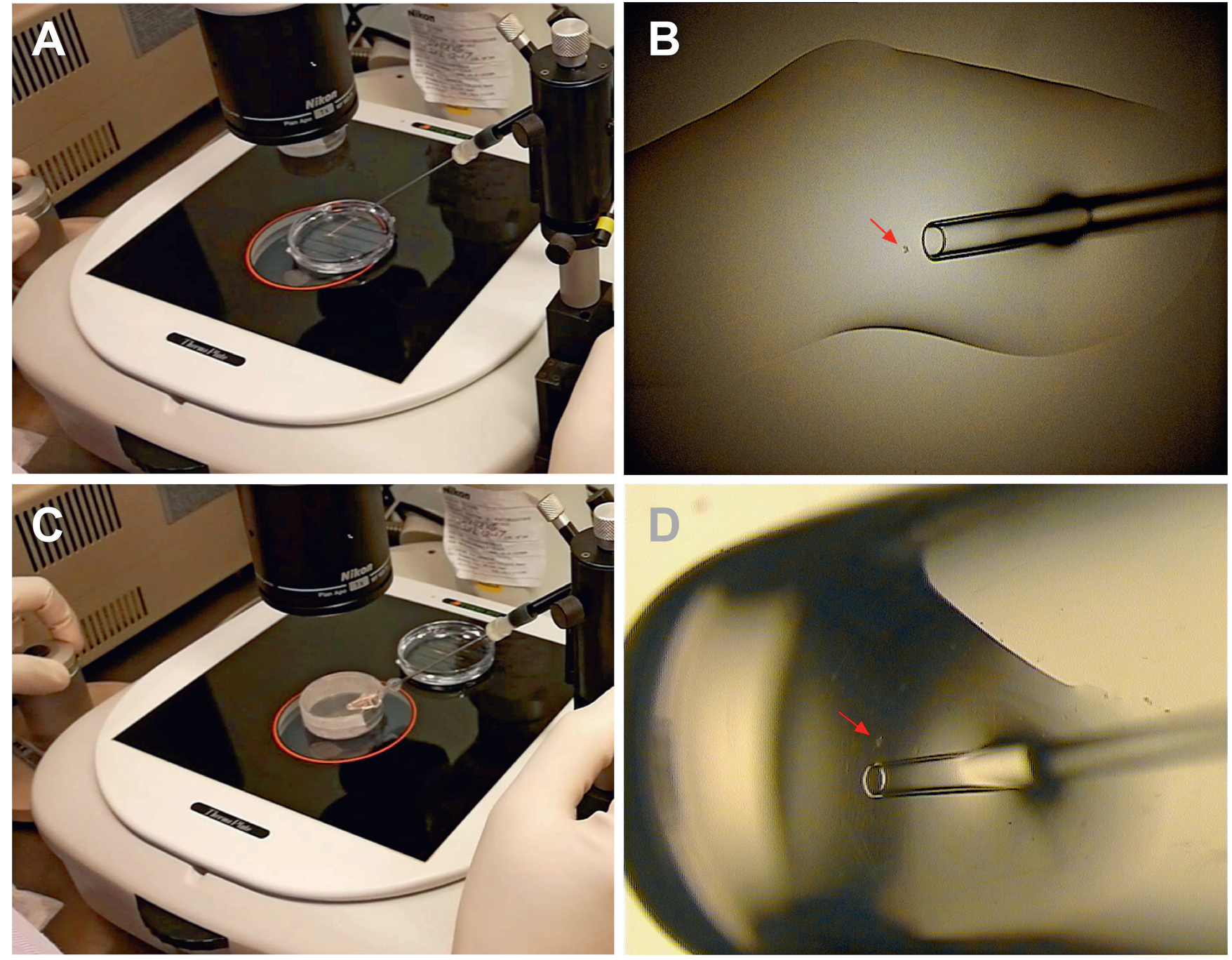

Fig. 3. Optimization of biopsy tubing procedural steps. A,B. The biopsy sample was transferred from biopsy dish. C,D. The locate the micropipette containing biopsy sample was inserted into the PCR tube, and the biopsy sample was expelled into the PCR tube. The red arrows showed the microscopic visualization of biopsy sample

for biopsy samples between December 2016 and December 2019 were included. The characteristics of the study patients are shown in Table 1. In laboratory outcomes, 2595 metaphase II (MII) oocytes were injected and cultured, 2113 were observed to have 2 pronuclear bodies and 2060 fertilized oocytes reached the cleavage stage. There were 1359 cleavage-stage embryos developed to blastocyst stage, of which 826 were usable blastocysts and 459 were highquality blastocysts. The fertilization rate, cleavage rate, blastocyst formation rate, usable blastocyst rate, and highquality blastocyst rate were $81.4 \%, 97.5 \%, 66.0 \%, 60.8 \%$, and $33.8 \%$, respectively (Table 2).

\section{PGT results and clinical outcomes of modified biopsy sample tubing method}

In the PGT results, of a total of 222 PGT cycles, 190 cycles achieved embryo biopsy, while 32 cycles (14.4\%) did not, as no embryo developed to the available blastocyst stage. Overall, 823 out of 826 (99.6\%) blastocyst biopsies resulted in successful amplification. Three $(0.4 \%)$, including 2 day 5 blastocysts (Fig. 4A,B) and 1 day 6 blastocyst (Fig. 4C) from different individual patients failed to amplify. The mean number of biopsied embryos per patient was $3.7 \pm 3.4$. Of the embryos successfully amplified, $100 \%$ had interpretable results and $38.2 \%$ were genetically transferable. The clinical pregnancy rate per transfer, live birth rate and miscarriage rate were $80.9 \%, 74.5 \%$ and $6.4 \%$, respectively (Table 2).

\section{Discussion}

A key caveat for PGT is the need to prevent the loss of biopsy samples during tubing. This problem is even more pronounced in patients from whom few oocytes are retrieved. How can the loss be minimized? Conventional PGT to date has focused on embryo biopsy and diagnosis, ${ }^{3,5,10}$ but not a single paper has been published focusing 
Table 1. Characteristics of the study population

\begin{tabular}{|c|c|}
\hline Indication & Total \\
\hline Number of patients, $n$ & 139 \\
\hline Number of cycles, n & 222 \\
\hline Maternal age $( \pm S D)$ [years] & $33.3 \pm 5.4$ \\
\hline BMI $\left[\mathrm{kg} / \mathrm{m}^{2}\right]$ & $21.7 \pm 2.6$ \\
\hline Infertility duration ( \pm SD) [years] & $2.3 \pm 2.5$ \\
\hline Basal FSH [IU/L] & $6.0 \pm 2.1$ \\
\hline Basal LH [IU/L] & $3.7 \pm 2.1$ \\
\hline \multicolumn{2}{|c|}{ Indication to PGT (\%) } \\
\hline PGT-A & $86 / 222(38.7)$ \\
\hline PGT-M & $54 / 222(24.3)$ \\
\hline PGT-SR & $78 / 222(35.1)$ \\
\hline Other & 4/222 (1.8) \\
\hline Retrieved oocytes, n (mean \pm SD) & $2087(14.5 \pm 8.9)$ \\
\hline Mll oocytes, n (mean \pm SD) & $1707(11.7 \pm 7.3)$ \\
\hline
\end{tabular}

BMI - body mass index; MII - metaphase II; PGT-A - PGT for aneuploidies; PGT-M - PGT for monogenic; PGT-SR - PGT for structural arrangements; SD - standard deviation.
Table 2. Embryological data and PGT results of modified biopsy sample transfer method

\begin{tabular}{|c|c|}
\hline Parameter & Total \\
\hline \multicolumn{2}{|l|}{ Cycle characteristics } \\
\hline Oocytes injected & 2595 \\
\hline Fertilization rate, n (\%) & $2113 / 2595(81.4)$ \\
\hline Cleavage rate, n (\%) & $2060 / 2113(97.5)$ \\
\hline Blastocyst formation rate, n (\%) & $1359 / 2060(66.0)$ \\
\hline Usable blastocyst rate, n (\%) & $826 / 1359(60.8)$ \\
\hline High-quality blastocyst rate, n (\%) & $459 / 1359(33.8)$ \\
\hline \multicolumn{2}{|l|}{ PGT results } \\
\hline Cycles with biopsiable embryos, n (\%) & 190/222 (85.6) \\
\hline Biopsied embryos, n (mean \pm SD) & $826(3.7 \pm 3.4)$ \\
\hline Amplification efficiency, n (\%) & $823 / 826(99.6)$ \\
\hline Not affected embryos diagnosed, n (\%) & $314 / 823(38.2)$ \\
\hline \multicolumn{2}{|l|}{ Clinical outcomes } \\
\hline Number of embryos transferred, $n$ (mean \pm SD) & $47(1.0 \pm 0.0)$ \\
\hline Implantation rate & 38/47 (80.9) \\
\hline Clinical pregnancy rate, n (\%) & 38/47 (80.9) \\
\hline Miscarriage rate & $3 / 47(6.4)$ \\
\hline Live birth rate (\% per ET) & $35 / 47(74.5)$ \\
\hline
\end{tabular}

SD - standard deviation; ET - embryo transfer.
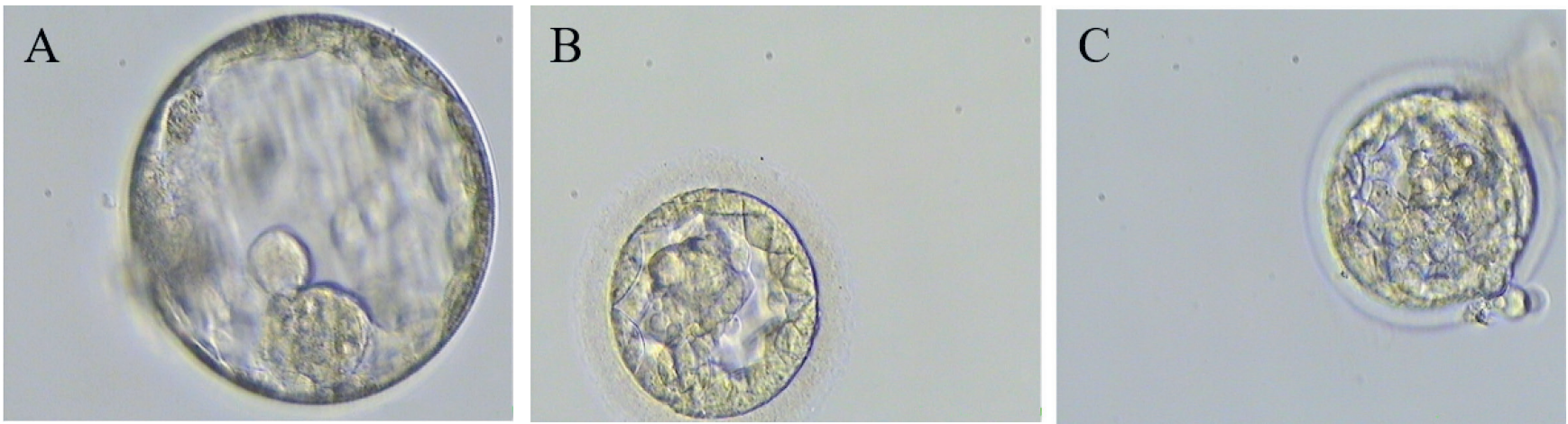

Fig. 4. Amplification failure - embryos from three different individual patients

on the optimization of the tubing method for placing biopsy samples into amplification tubes for PGT. Our center had started to advance the safety and accuracy of tubing and to encourage this technique to be adopted into clinical practice in order to improve PGT.

Precise control is required during tubing, because of the size of the biopsy samples (no more than $30 \mu \mathrm{m}$ ) and the volume of lysis buffer in the amplification tube (usually $3 \mu \mathrm{L}$ in whole genome amplification). However, the manual control of micropipettes is notoriously imprecise. Therefore, tubing biopsy samples into PCR tubes is a challenging task for many embryologists. For European Society for Human Reproduction and Embryology (ESHRE) data collection XIV-XV, ${ }^{16} 71$ centers reported data on 11,481 cycles with PGT, along with 64,395 biopsies from January 2011 to December 2012; DNA amplification products were detected in 63,388 biopsies (98.4\%) and 59,556 biopsies ( $92.5 \%$ ) had interpretable results. In a study by Capalbo et al. on 2586 blastocyst-stage biopsies from 906 IVF-PGT cycles at 3 IVF centers between April 2013 and December 2014, the amplification success rate and diagnostic efficiency were $98.8 \%(2556 / 2586)$ and $94.2 \%$ $(2,437 / 2,586)$, respectively. ${ }^{17}$ More recently, a study that included 8990 blastocyst-stage biopsies of 6 IVF centers found that $98.0 \%$ of biopsies could be detected through the presence of DNA after amplification, but up to $97.5 \%$ of biopsied samples were able to be conclusively diagnosed. ${ }^{18}$ This evidence suggests that diagnostic efficiency is continually improving, whereas the amplification success rate has remained unchanged. This means that with new genetic testing technology, the accuracy and sensitivity of the analysis method has increased, ${ }^{19}$ though 
no significant development has been made in the tubing or DNA amplification techniques.

In the present study, the amplification success rate was $99.6 \%$, which was higher than the reference studies selected in it. The reasons for the outcomes were the proposed optimization approach, which included a few improvements: the modified micromanipulator aided in the fine movement of the micropipette, the modified microinjector minimized the amount of medium co-transferred with the cell, and the modified transfer device combined with a stereomicroscope provided a vision-based tubing system. These simple solutions can lead to a reduced need for an embryo re-biopsy, benefiting the amplification success rate and resulting in improved PGT results. As is wellknown, the guidelines of the Preimplantation Genetic Diagnosis International Society and the ESHRE PGD consortium all recommend that the amplification success rate be no less than $90 \% .^{20,21}$ Based on data from surveys and the PGD Consortium, the Vienna consensus recommends that the reference values of a successful biopsy rate be as follows: competency $\geq 90 \%$ and benchmark $\geq 95 \%$. Additionally, the benchmark of a tubing rate of $95 \%$ was reported in the Alpha survey. ${ }^{22}$ These recommendations confirm that this modified approach is technically solid. At the same time, the tubing procedure with the modified method was easily standardized among the operators. As the success of a PGT treatment cycle is the result of great attention to detail, the optimization of any procedural steps is a pragmatic goal. ${ }^{8}$

A common characteristic between all biopsy stages is the limited quantity of samples available for genetic analysis, potentially compounded by the often sub-optimal quality of the embryo cell biopsied. ${ }^{23}$ The cell death in TE cells, damage to genomic DNA from the laser or mechanical injury during the biopsy may also affect the quality of the biopsy samples. ${ }^{24,25}$ Damaged DNA from poorquality biopsy samples may lead to an amplification failure or to low-confidence profiles. ${ }^{26}$ In the present study, 3 biopsies did not produce any DNA amplification (Fig. 4). Amplification failure (AF) suggests only the absence of TE cells in the test tube or the presence of degenerated/lysed cells. The rate of AF is mainly imputable to technical aspects, and an increasing trend has been found from the most to the least experienced clinics. ${ }^{18}$ As the control was visual and precise in this study, AF may be related not to the loss of the biopsied TE cells during the transfer from the biopsy dish to the amplification tube, but to the biopsy procedure. In fact, careful consideration is required when selecting the optimal time to perform biopsy for PGT. According to our results, as the expansion grade of blastocysts in 3 biopsies of AF was low, these blastocysts would be better with extended culturing.

In this study, biopsy and embryo transfer were performed at the blastocyst stage. The different days of biopsy turned out to be the main variable affecting both the presence and quality of the analyzed DNA. From days 3 to 5, longer culturing in vitro and wider blastocyst expansion were associated with smaller biopsy samples (i.e., with a higher DNA content and quality). ${ }^{18}$ Also, the evidence suggests that cleavage-stage biopsy impairs the potential for human embryo implantation significantly more than blastocyst biopsy, ${ }^{27-29}$ and embryo transfer at the blastocyst stage is accompanied by an improvement in pregnancy rates. From recently performed reviews and meta-analyses, the live birth rate of PGT ranged between $30 \%$ and $70 \% .{ }^{30-32}$ In the present study, the implantation rate, clinical pregnancy rate, live birth rate, and miscarriage rate were $80.9 \%$, $80.9 \%, 74.5 \%$, and $6.4 \%$, respectively. These findings suggest that the PGT technique in our IVF center is stable.

The study has a few limitations. Because there is no ideal indicator for tubing, our outcome measure was the amplification success rate rather than the tubing rate. No comparative studies between the conventional method and the modified approach were performed in the same lab, and there are only few reports on amplification success rate. However, for safety assessment and training, the modified approach was evaluated both in a mouse experimental system and in a preclinical study using spare embryos from routine IVF procedures, before being applied in clinical PGT. The results were shown to be compatible with a high tubing rate of biopsy samples.

\section{Conclusions}

The present study demonstrates that the tubing method of biopsy samples can be a viable option for clinical application in PGT cycles. Instead of manual control, which requires many procedures of training to obtain proficiency, this tubing procedure is simple to perform and has a high degree of precision.

\section{ORCID iDs}

Shuai Liu (1) https://orcid.org/0000-0003-4864-5804 Zhiheng Chen (1) https://orcid.org/0000-0001-7421-4042 Yunhao Liang (1) https://orcid.org/0000-0001-9314-1694

Hui Wang (1) https://orcid.org/0000-0002-1361-0030

Li Yang (1) https://orcid.org/0000-0003-3379-2553

Ling Sun (1) https://orcid.org/0000-0002-4267-8210

\section{References}

1. Harper JC, Wilton L, Traeger-Synodinos J, et al. The ESHRE PGD Consortium: 10 years of data collection. Hum Reprod Update. 2012;18(3): 234-247.

2. Kakourou G, Kahraman S, Ekmekci GC, et al. The clinical utility of PGD with HLA matching: A collaborative multi-centre ESHRE study. Hum Reprod. 2018;33(3):520-530.

3. Ubaldi FM, Cimadomo D, Capalbo A, et al. Preimplantation genetic diagnosis for aneuploidy testing in women older than 44 years: A multicenter experience. Fertil Steril. 2017;107(5):1173-1180.

4. Rubio C, Bellver J, Rodrigo L, et al. In vitro fertilization with preimplantation genetic diagnosis for aneuploidies in advanced maternal age: A randomized, controlled study. Fertil Steril. 2017;107(5):1122-1129.

5. Cimadomo D, Ubaldi FM, Capalbo A, et al. Failure mode and effects analysis of witnessing protocols for ensuring traceability during PGD/PGS cycles. Reprod Biomed Online. 2016;33(3):360-369. 
6. Preimplantation Genetic Diagnosis International Society (PGDIS). Guidelines for good practice in PGD: Programme requirements and laboratory quality assurance. Reprod Biomed Online. 2008;16(1):134-147.

7. Harton GL, Magli MC, Lundin K, Montag M, Lemmen J, Harper JC; European Society for Human Reproduction and Embryology (ESHRE) PGD Consortium/Embryology Special Interest Group. ESHRE PGD Consortium/Embryology Special Interest Group: Best practice guidelines for polar body and embryo biopsy for preimplantation genetic diagnosis/screening (PGD/PGS). Hum Reprod. 2011;26(1):41-46.

8. Harper JC, Sengupta S, Vesela K, et al. Accreditation of the PGD laboratory. Hum Reprod. 2010;25(4):1051-1065.

9. Imudia AN, Plosker S. The past, present, and future of preimplantation genetic testing. Clin Lab Med. 2016;36(2):385-399.

10. Harton GL, De Rycke M, Fiorentino F, et al; European Society for Human Reproduction and Embryology (ESHRE) PGD Consortium. ESHRE PGD consortium best practice guidelines for amplificationbased PGD. Hum Reprod. 2011;26(1):33-40.

11. Malter HE. Micromanipulation in assisted reproductive technology. Reprod Biomed Online. 2016;32(4):339-347.

12. Shojaei-Baghini E, Zheng Y, Sun Y. Automated micropipette aspiration of single cells. Ann Biomed Eng. 2013;41(6):1208-1216.

13. Zhang X, Leung C, Lu Z, Esfandiari N, Casper RF, Sun Y. Controlled aspiration and positioning of biological cells in a micropipette. IEEE Trans Biomed Eng. 2012;59(4):1032-1040.

14. Banerjee AG, Gupta SK. Research in automated planning and control for micromanipulation. IEEE Transactions on Automation Science and Engineering. 2013;10(3):485-495.

15. Gardner DK, Schoolcraft WB. In-vitro culture of human blastocyst In: Jansen R, Mortimer D, eds. Towards Reproductive Certainty: Infertility Genetics Beyond 1999: The Plenary Proceedings of the $11^{\text {th }}$ World Congress. New York NY: Parthenon Press; 1999:378-388.

16. De Rycke M, Goossens V, Kokkali G, Meijer-Hoogeveen M, Coonen E, Moutou C. ESHRE PGD Consortium data collection XIV-XV: Cycles from January 2011 to December 2012 with pregnancy follow-up to October 2013. Hum Reprod. 2017;32(10):1974-1994.

17. Capalbo A, Ubaldi FM, Cimadomo D, et al. Consistent and reproducible outcomes of blastocyst biopsy and aneuploidy screening across different biopsy practitioners: A multicentre study involving 2586 embryo biopsies. Hum Reprod. 2016;31(1):199-208.

18. Cimadomo D, Rienzi L, Romanelli V, et al. Inconclusive chromosomal assessment after blastocyst biopsy: Prevalence, causative factors and outcomes after re-biopsy and re-vitrification. A multicenter experience. Hum Reprod. 2018;33(10):1839-1846.

19. Xu K, Montag M. New perspectives on embryo biopsy: Not how, but when and why? Semin Reprod Med. 2012;30(4):259-266.
20. Thornhill AR, deDie-Smulders CE, Geraedts JP, et al; ESHRE PGD Consortium. ESHRE PGD Consortium "Best practice guidelines for clinical preimplantation genetic diagnosis (PGD) and preimplantation genetic screening (PGS)". Hum Reprod. 2005;20(1):35-48.

21. Preimplantation Genetic Diagnosis International Society. The Preimplantation Genetic Diagnosis International Society (PGDIS): Guidelines for good practice in PGD. Reprod Biomed Online. 2004;9(4): 430-434.

22. ESHRE Special Interest Group of Embryology and Alpha Scientists in Reproductive Medicine. The Vienna consensus: Report of an expert meeting on the development of art laboratory performance indicators. Hum Reprod Open. 2017;35(5):494-510.

23. Dreesen J, Destouni A, Kourlaba G, et al. Evaluation of PCR-based preimplantation genetic diagnosis applied to monogenic diseases: A collaborative ESHRE PGD consortium study. Eur J Hum Genet. 2014;22(8):1012-1018.

24. Zhang S, Tan K, Gong F, et al. Blastocysts can be rebiopsied for preimplantation genetic diagnosis and screening. Fertil Steril. 2014;102(6): 1641-1645.

25. Wininger JD, Taylor TH, Orris JJ, Glassner M, Anderson SH. Pregnancy after rebiopsy and vitrification of blastocysts following allele dropout after day 3 biopsy. Fertil Steril. 2011;95(3):1122.e1-e2.

26. Parriego M, Coll L, Vidal F, et al. Inconclusive results in preimplantation genetic testing: Go for a second biopsy? Gynecol Endocrinol. 2019;35(1):90-92.

27. Sciorio R, Tramontano L, Catt J. Preimplantation genetic diagnosis (PGD) and genetic testing for aneuploidy (PGT-A): Status and future challenges. Gynecol Endocrinol. 2020;36(1):6-11.

28. Jing $\mathrm{S}$, Luo K, He H, et al. Obstetric and neonatal outcomes in blastocyst-stage biopsy with frozen embryo transfer and cleavage-stage biopsy with fresh embryo transfer after preimplantation genetic diagnosis/screening. Fertil Steril. 2016;106(1):105-112.e4.

29. Scott RT Jr, Upham KM, Forman EJ, Zhao T, Treff NR. Cleavage-stage biopsy significantly impairs human embryonic implantation potential while blastocyst biopsy does not: A randomized and paired clinical trial. Fertil Steril. 2013;100(3):624-630.

30. Wei D, Liu JY, Sun Y, et al. Frozen versus fresh single blastocyst transfer in ovulatory women: A multicentre, randomised controlled trial. Lancet. 2019;393(10178):1310-1318.

31. Zeng M, Su S, Li L. Comparison of pregnancy outcomes after vitrification at the cleavage and blastocyst stage: A meta-analysis. J Assist Reprod Genet. 2018;35(1):127-134.

32. Martins WP, Nastri CO, Rienzi L, van der Poel SZ, Gracia C, Racowsky C. Blastocyst vs cleavage-stage embryo transfer: Systematic review and meta-analysis of reproductive outcomes. Ultrasound Obstet Gynecol. 2017;49(5):583-591. 